\title{
Clinical characteristics and pathogenesis of cerebellar glioblastoma
}

\author{
YOSHINOBU TAKAHASHI $^{1,2}$, KEISHI MAKINO ${ }^{1}$, HIDEO NAKAMURA $^{1}$, TAKUICHIRO HIDE ${ }^{1}$, \\ SHIGETOSHI YANO $^{1}$, HAJIME KAMADA ${ }^{2}$ and JUN-ICHI KURATSU ${ }^{1}$ \\ ${ }^{1}$ Department of Neurosurgery, Graduate School of Medical Science, Kumamoto University, Kumamoto 860-8556; \\ ${ }^{2}$ Department of Neurosurgery, Hokuto Hospital, Obihiro, Hokkaido 080-0039, Japan
}

Received November 11, 2013; Accepted May 25, 2014

DOI: $10.3892 / \mathrm{mmr} .2014 .2549$

\begin{abstract}
Cerebellar glioblastomas (GBMs) are rare, with neither their pathogenesis nor prognosis being completely understood. The present study aimed to clarify the clinical characteristics of cerebellar GBMs by comparison with supratentorial GBMs, focusing particularly on the pathogenesis. The clinical factors between cerebellar $(n=10)$ and supratentorial $(n=216)$ GBMs were compared. Additionally, p53 and epidermal growth factor receptor (EGFR) levels were investigated in six patients by immunostaining as well as the isocitrate dehydrogenase 1 (IDH1) status of five patients by direct sequencing. Eight males and two females participated in the present study, the mean age at diagnosis was 56.6 years and the range $37-75$ years. Four patients presented with hydrocephalus and one with brainstem involvement, and two patients were diagnosed with neurofibromatosis type 1 . Two patients had previously received radiotherapy, eight patients received postoperative radiotherapy and seven chemotherapy. The mean Karnofsky performance status (KPS) score was lower in patients with cerebellar GBMs compared to those with supratentorial GBM; however, the survival times did not differ between the two groups. All of the cases of six cerebellar GBMs were p53-positive and EGFR-negative, as detected by immunostaining, consistent with secondary GBM. However, no IDH1 mutations were detected in any of the five cases of cerebellar GBMs analyzed, indicating that these tumors were not of the secondary type. The KPS score with cerebellar GBMs may be lower due to hydrocephalus, which was ameliorated by surgery but may have impacted the survival rate. It was confirmed that cerebellar GBMs were identical to supratentorial GBMs with respect to its clinical features, with the possible exception of the KPS score. The
\end{abstract}

Correspondence to: Dr Yoshinobu Takahashi, Department of Neurosurgery, Hokuto Hospital, 7-5 Inada, Obihiro, Hokkaido 080-0039, Japan

E-mail: yosinobu@hokuto7.or.jp

Key words: cerebellar, glioblastoma, p53, epidermal growth factor receptor, isocitrate dehydrogenase 1 , hydrocephalus present study's genetic analyses indicated that cerebellar GBMs may develop via a pathway different from that of either primary or secondary GBM.

\section{Introduction}

Glioblastoma (GBM) is the most common type of malignant brain tumor. However, cerebellar GBMs are rare and accounts for $0.4-3.4 \%$ of all GBM cases (1-3). Cerebellar GBMs are not yet completely understood in terms of prognosis, due to their rarity. The present study aimed to clarify the clinical characteristics of cerebellar GBMs by comparison with supratentorial GBMs. GBMs may develop de novo (primary type) or from previous low grade astrocytomas (secondary type) $(4,5)$. Secondary GBMs often contain a p53 mutation (65\%), while primary GBMs are generally characterized by the absence of heterozygosity $10 \mathrm{q}$, epidermal growth factor (EGFR) amplification (36\%) and p53 mutation at a frequency lower than $30 \%$ (5). The isocitrate dehydrogenase 1 (IDH1) mutation may be highly a selective molecular marker for secondary GBMs that complement clinical criteria to distinguish them from primary GBMs (6). Furthermore, the present study aimed to focus on the pathogenesis of cerebellar GBMs.

\section{Materials and methods}

Cerebellar GBMs $(n=10)$ were compared with supratentorial $(n=216)$ GBMs with respect to clinical factors [gender, age, Karnofsky performance status (KPS) score, extent of surgical resection and survival time]. In addition, p53 and EGFR levels were investigated in six patients by immunostaining and the IDH1 status of five patients by direct sequencing. The present study was approved by the ethics committee of Kumamoto University Hospital, Kumamoto, Japan). Written informed consent was obtained from the patients and/or their legal guardians.

Immunostaining. Surgical specimens were fixed, embedded in paraffin and $4-\mu \mathrm{m}$ sections were prepared. The sections were deparaffinized in xylene and rehydrated in a graded ethanol-to-water series. Endogeneous peroxidase activity was blocked with hydrogen peroxidase. Immunostaining was performed using the avidin-biotinylated enzyme 
Table I. Clinical characteristics of glioblastomas in the cerebellum and in the cerebral hemisphere.

\begin{tabular}{|c|c|c|c|c|}
\hline Factors & Characteristics & Cerebellar & Cerebral & P-value \\
\hline Cases (n) & & 10 & 226 & \\
\hline \multirow[t]{2}{*}{ Gender } & Male & 8 & 134 & NS \\
\hline & Female & 2 & 92 & \\
\hline Mean age (years) & & 56.6 & 58.1 & NS \\
\hline Mean KPS & & 68.0 & 80.4 & 0.03 \\
\hline \multirow[t]{2}{*}{ Surgery } & Total & 2 & 89 & NS \\
\hline & Partial or biopsy & 8 & 137 & \\
\hline Median survival (months) & & 9 & 12 & NS \\
\hline
\end{tabular}

KPS, Karnofsky performance status. NS, not significant.

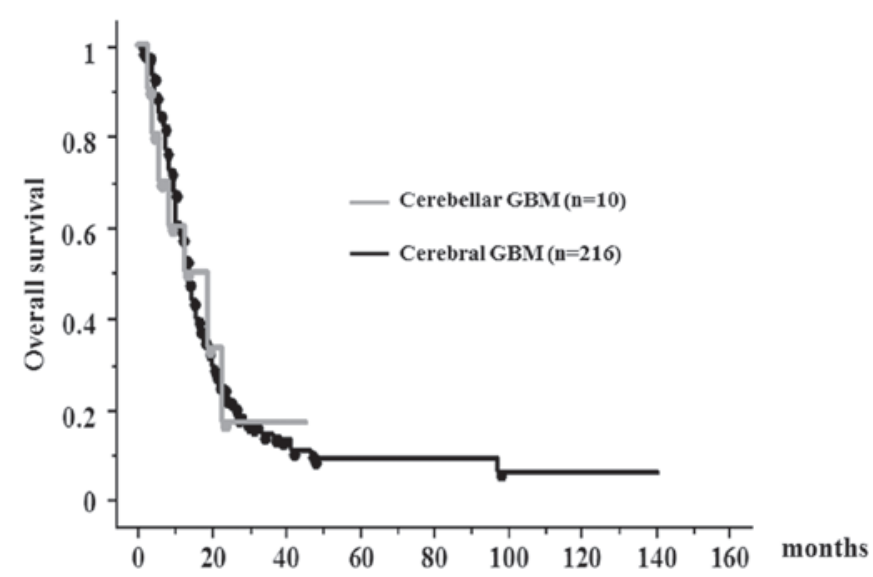

Figure 1. No significant difference in the survival time between cerebellar and supratentorial GBM patients was identified. GBM, glioblastomas.

complex (ABC) method (VECTASTAIN ABC kit; Vector Laboratories, Burlingame, CA, USA). The following primary antibodies were used: Anti-glial fibrillary acidic protein (GFAP; Dako, Tokyo, Japan), anti-MIB-1 (Dako), antip53 (Do-7, Dako) and anti-EGFR (EGFR113; Novocastra Laboratories Ltd., Milton Keynes, UK).

Direct DNA sequencing of IDH1 mutations. Genomic DNA was isolated from the surgical specimens using the Qiagen DNA Mini kit (Qiagen, Valencia, CA, USA). The polymerase chain reaction (PCR) primers for the genomic region corresponding to the IDH1 exon 4 that encodes the codon R132 were as follows: IDH1 sense, 5'-AAACAAATGTGGAAATCACC-3' and antisense, 5'-TGCCAACATGACTTACTTGA-3'. The PCR conditions were: $94^{\circ} \mathrm{C}$ for $5 \mathrm{~min}, 36$ cycles at $94^{\circ} \mathrm{C}$ for $30 \mathrm{sec}$, $55^{\circ} \mathrm{C}$ for $30 \mathrm{sec}$ and $72^{\circ} \mathrm{C}$ for $1 \mathrm{~min}$, followed by an extension at $72^{\circ} \mathrm{C}$ for $5 \mathrm{~min}$. PCR was performed using Ex-Taq high-sensitivity DNA polymerase (Takara Bio, Shiga, Japan). The PCR products were purified using the QIAquick PCR purification kit (Qiagen Valencia, CA, USA) according to the manufacturer's instructions. Sequencing reactions were performed using the primers mentioned above and a Big Dye Terminator Cycle Sequencing kit (PE Applied Biosystems, Foster City, CA, USA) on an ABI377 automated sequencer (PE Applied Biosystems).
Summary of cases. The characteristics of the patients with cerebellar GBMs are described in Table I. There was no clinical or histological evidence of a pre-existing, less malignant precursor lesion in all of the cases. Of the 10 patients, six (patients 1, 2, 3, 4, 5, 6) were treated at Kumamoto University Hospital between 1997 and 2010. During this time, 216 patients with supratentorial GBMs were also treated. In this series of eight males and two females, the mean age at diagnosis was 56.6 years and the range was 37-75 years. The average KPS score was 68.0. A total of four patients suffered from hydrocephalus, one had brainstem involvement and two patients had neurofibromatosis type 1 . Two patients had previously received radiotherapy, one for a medulloblastoma 34 years earlier, and the other for a hemangioblastoma 15 years earlier. Two patients underwent macroscopic total resection, the other eight partial resection and eight patients received postoperative radiotherapy and seven chemotherapy. In total, seven patients died at 3, 4, 6, 9, 13, 19 and 23 months, respectively, following surgical resection. Three patients were alive as of August 2011.

\section{Results}

Clinical characteristics and survival times of cerebellar GBM patients. Only the mean KPS score was lower in patients with cerebellar GBMs compared to those with supratentorial GBMs (Table I). No significant difference was noted in survival time between the cerebellar and supratentorial GBM patients (Fig. 1).

p53 and EGFR immunostaining and IDH1 status in cerebellar GBMs. All of the six cerebellar GBMs were p53-positive and EGFR-negative by immunostaining. Additionally, no IDH1 mutations were detected in any of the five cerebellar GBMs analyzed (Table II).

\section{Illustrative patient}

Patient six. In April 2010, a 45-year-old man presented with nausea, vomiting and gait disturbance. The patient had been diagnosed with neurofibromatosis type 1 by a dermatologist five years earlier. A neurological examination revealed cerebellar ataxia and magnetic resonance (MR) imaging revealed a $35 \mathrm{~mm}$ in diameter tumor in the cerebellar vermis. The tumor had relatively defined borders, demonstrated mild peritumoral edema and was heterogeneously enhanced with gadolinium 
Table II. Clinical data of 10 patients with cerebellar glioblastoma.

\begin{tabular}{|c|c|c|c|c|c|c|c|c|c|}
\hline Case & $\begin{array}{l}\text { Age } \\
\text { (years) }\end{array}$ & Jender & Location & Hydrocephalus & $\begin{array}{l}\text { Brain stem } \\
\text { invasion }\end{array}$ & Surgery & IDH1 & $\begin{array}{l}\text { Survival } \\
\text { (months) }\end{array}$ & $\begin{array}{l}\text { Other } \\
\text { Findings }\end{array}$ \\
\hline 1 & 51 & $\mathrm{~F}$ & Rt hemisphere & $(-)$ & $(-)$ & partial & wild & 23 & \\
\hline 2 & 75 & M & $\begin{array}{l}\text { Lt hemisphere, } \\
\text { vermis }\end{array}$ & $(+)$ & $(-)$ & partial & wild & 9 & \\
\hline 3 & 41 & M & $\begin{array}{l}\text { Lt hemisphere, } \\
\text { vermis }\end{array}$ & $(+)$ & $(-)$ & partial & wild & 6 & radiation induced \\
\hline 4 & 50 & M & $\begin{array}{l}\text { Rt hemisphere, } \\
\text { vermis }\end{array}$ & $(+)$ & $(-)$ & partial & not investigated & alive & NF-1 \\
\hline 5 & 60 & M & Rt hemisphere & $(-)$ & $(-)$ & partial & wild & alive & \\
\hline 6 & 45 & M & vermis & $(-)$ & $(-)$ & total & wild & alive & NF-1 \\
\hline 7 & 71 & M & vermis & $(+)$ & $(+)$ & partial & not investigated & 3 & \\
\hline 8 & 37 & M & vermis & $(-)$ & $(-)$ & partial & not investigated & 4 & radiation induced \\
\hline 9 & 68 & $\mathrm{~F}$ & Lt hemisphere & $(-)$ & $(-)$ & total & not investigated & 19 & \\
\hline 10 & 68 & M & $\begin{array}{l}\text { Rt hemisphere, } \\
\text { vermis }\end{array}$ & $(-)$ & $(-)$ & partial & not investigated & 13 & \\
\hline
\end{tabular}

Rt, right; Lt, left; IDH, isocitrate dehydrogenase; NF-1, neurofibromatosis type1; M, male; F, female.
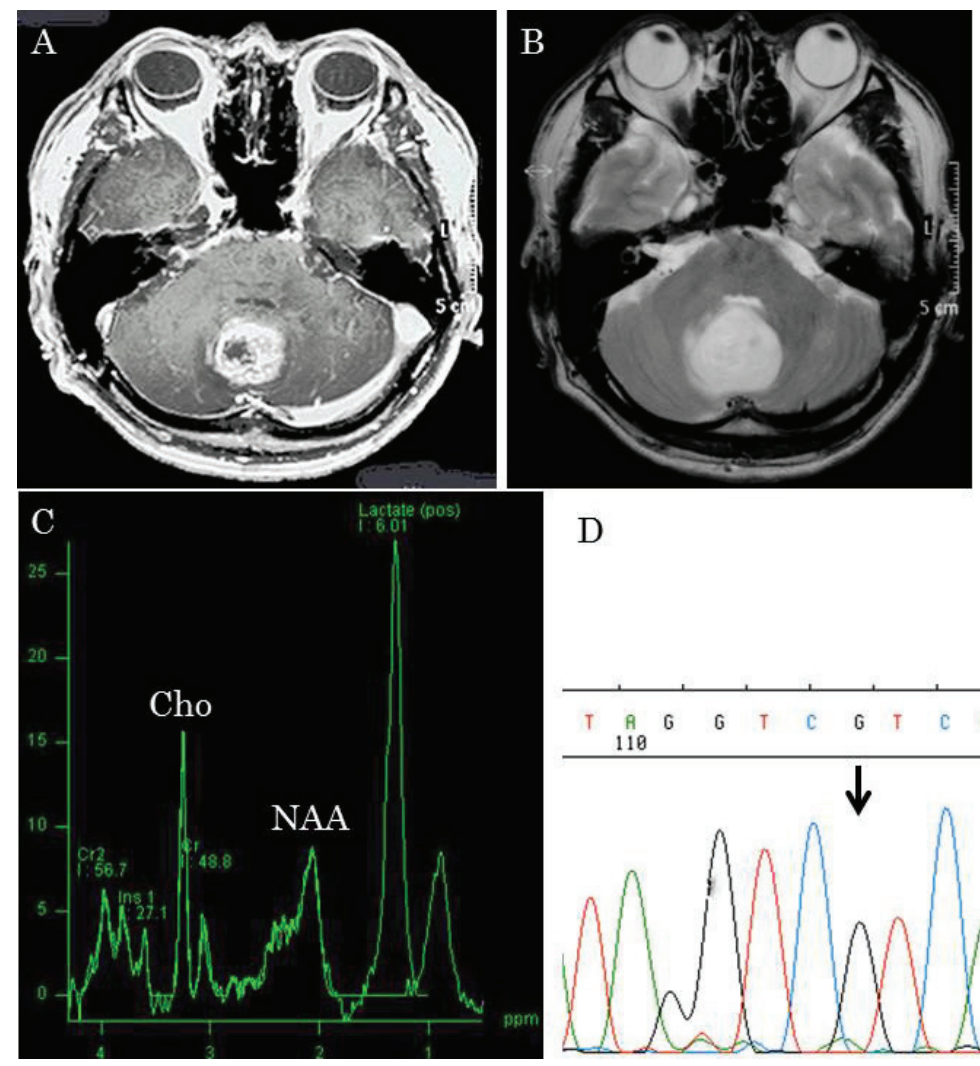

$\mathrm{D}$

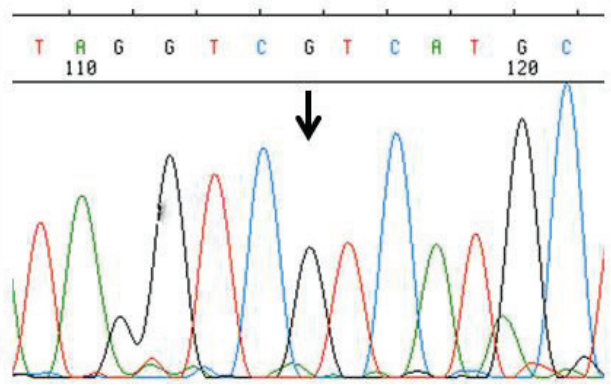

Figure 2. (A) T1-weighted magnetic resonance (MR) imaging with Gd-DTPA revealed a heterogeneously enhanced lesion in the cerebellar vermis. (B) T2-weighted MR imaging demonstrated that the mass had relatively defined borders with mild peritumoral edema. (C) MR spectroscopy of the lesion demonstrated a high choline peak in contrast to the decreased $N$-acetyl-aspartate peak observed with high-grade tumors. (D) The wild-type of the IDH1 gene was detected by direct sequencing. (Arrow indicates codon 132). Gd-DTPA, gadolinium diethylene triamine pentaacetic acid; MR, magnetic resonance.

(Fig. 2A and B). MR spectroscopy of the lesion demonstrated a high choline peak in contrast to a decreased $N$-acetyl-aspartate peak (Fig. 2C). A total tumor resection via a suboccipital craniotomy was performed. A histological examination revealed
GBM. Tumor cells were spindle-shaped with irregular, moderately pleomorphic nuclei (Fig. 3A and B). The tumor cell cytoplasm was positive for GFAP (Fig. 3C) and $20 \%$ of the cell nuclei were positive for the MIB1 gene (Fig. 3D). The 

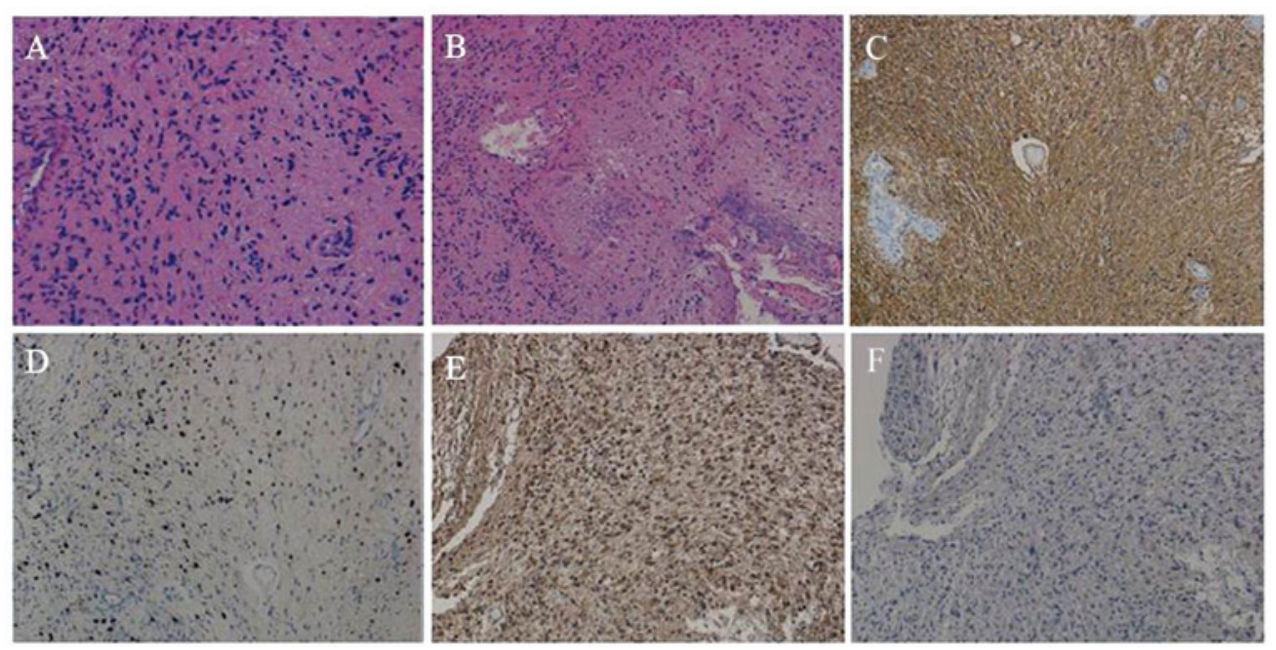

Figure 3. (A) Histological examination of the excised specimens revealed a cellular tumor composed of spindle-shaped cells with irregular, moderately pleomorphic nuclei (H\&E staining). (B) Pseudo-palisading necrosis was present (H\&E staining). (C) The tumor cells revealed cytoplasmic positivity for glial fibrillary acidic protein. (D) In total, $20 \%$ of the nuclei were positive for MIB1. (E) The tumor cells were diffusely immunoreactive for p53. (F) None of the tumor cells were immunoreactive for EGFR. magnification, x200. H\&E, hematoxylin and eosin; EGFR, epidermal growth factor receptor.

tumor cells were diffusely immunoreactive for p53 (Fig. 3E), but none were immunoreactive for EGFR (Fig. 3F). Wild-type IDH1 was identified by direct sequencing (Fig. 2D). The patient received radiotherapy with concomitant and adjuvant temozolomide chemotherapy and there was no evidence of recurrence during the subsequent 16-month follow-up.

\section{Discussion}

Cerebellar GBMs are a rare condition. Reports on cerebellar GBMs have described between one to seven cases and there are only a few series analyzing more than 10 cerebellar GBMs $(7,8)$. Its rarity hampers the identification of the clinical features and biology of this tumor, as compared with the well-understood supratentorial GBMs. The present study examined a total of 10 cases of cerebellar GBMs, of which six cases were identified at Kumamoto University Hospital and four cases at affiliated hospitals. Cerebellar GBMs were compared with supratentorial GBMs, focusing on clinical features.

Clinical factors other than the KPS score did not differ between the patients with these two types of GBMs. The KPS score with cerebellar GBMs may have been lower due to hydrocephalus. Hydrocephalus was ameliorated by surgery, which may have affected the survival time. In the present study, no significant difference was noted in the survival time between patients with cerebellar those with supratentorial GBM. Weber et al (7) reviewed 45 patients with cerebellar GBMs treated between 1979 and 2004. It was suggested that the prognosis of cerebellar GBM patients appeared to be identical to that of cases with supratentorial tumors. Additionally, the authors also concluded that brain stem invasion was an adverse prognostic factor. In the present study one of the cases presented with brain stem invasion and this patient had the shortest survival time.

At Kumamoto University Hospital, six cerebellar GBMs (2.6\%) were identified among 232 GBMs. The reason for the low frequency of cerebellar GBMs has not yet been elucidated. Of note, among the 10 cerebellar GBM cases, there were two neurofibromatosis type 1 (NF-1) patients and two patients who had received radiation therapy several years earlier. It was speculated that cerebellar GBMs may develop only in the setting of major prior genetic mutation.

NF-1 is one of the most common inherited autosomal dominant genetic disorders in humans, with frequencies ranging from 1 in 2,600 to 1 in 3,000 $(9,10)$. This disease carries an increased risk of developing benign and malignant tumors of the central and peripheral nervous systems. The majority of intracranial neoplasms in children with NF-1 are thought to be pathologically consistent with pilocytic astrocytomas (PA). A number of PAs in the setting of NF-1 may arrest spontaneously and do not require intervention. Several cases with spontaneous malignant transformation of PA have been reported (11-14). However, the specimens used in the present study did not feature any PA component. The NF-1 gene located on chromosome 17q11.2, which produces neurofibromin, has a role in cell signaling $(15,16)$. Mouse strains that lack p53 and harbor a conditional allele of the NF-1 tumor suppressor gene, resulting in negative regulation of Ras, develop malignant astrocytomas that demonstrate histological features of GBM (17). Radiationinduced GBMs are a rare complication of radiotherapy. Since the first radiation-induced GBM case was described in 1978 by Klériga et al (18), a number of such gliomas have been reported (19-25). The types of radiation-induced malignant glioma were GBMs in 69 (75\%) and anaplastic astrocytoma in $23(25 \%)$ out of 92 patients reported by Paulino et al (26). It has been suggested that radiation-induced neoplasms result from insufficient repair of ionizing radiation-induced DNA strand breaks in tumor suppressor genes or proto-oncogenes. Paulino et al (26) suggested that radiation-induced GBMs may be a type of secondary GBM, based on molecular genetic analyses showing p53 mutations but no EGFR amplification in these tumors. In both GBMs following NF-1 and radiation-induced GBM, p53 may have a significant role in tumorigenesis.

Returning to the original topic of cerebellar GBM, Utsuki et al (27) reported that all four cerebellar GBMs they 
encountered were p53-positive and EGFR-negative by immunostaining. Saito et al (28) identified that all of the seven cerebellar GBMs they examined were EGFR-negative, and six out of the seven were p53-positive. Akimoto et al (29) reported four cerebellar GBMs, three of which were p53-positive. GBMs may develop de novo (primary GBMs) or via progression from low-grade or anaplastic astrocytoma (secondary GBMs). Primary GBMs develop in older patients and typically demonstrate genetic alterations (EGFR amplification, p16/INK4a deletion and/or phosphatase and tensin homolog mutations) at frequencies of $24-34 \%$. Secondary GBMs develop in younger patients and frequently overexpress platelet-derived growth factor and cyclin-dependent kinase 4 as well as p53 mutations (65\%) and loss of retinoblastoma protein are observed, all of which have major roles in such transformations (4). Immunostaining results indicated cerebellar GBMs to be of the secondary type. In the present study, immunostaining yielded the same result. Recently, a large-scale verification study revealed IDH1 mutations in $50-80 \%$ of patients with grade 2 astrocytomas, oligodendrogliomas or secondary GBMs, whereas IDH1 mutations were rare in patients with primary GBMs $(6,30-37)$. If cerebellar GBMs are of the secondary type, a high frequency of IDH1 mutation would be expected. The IDH1 status of five cerebellar GBMs was investigated by direct sequencing. The wild-type of the IDH1 gene was detected in all five cases. In addition, Utsuki et al (27) detected wild-type IDH1 in all four cerebellar GBMs they examined by immunostaining. These results contradict the hypothesis that cerebellar GBMs are of the secondary type. Taking these observations together with the rarity of cerebellar GBM, it is reasonable to suggest that there are additional pathways underlying the development of cerebellar GBM, different from those leading to either primary or secondary GBM. Therefore, it is apparent that further analyses of a larger number of patients are necessary.

In conclusion, the present study confirmed cerebellar and supratentorial GBMs to essentially be identical with respect to clinical features. Furthermore, it was suggested that cerebellar GBMs may develop via pathways different from those of either primary or secondary GBM.

\section{Acknowledgements}

The authors would like to thank Mrs. Masayo Obata (Department of Neurosurgery, Graduate School of Medical Science, Kumamoto University, Kumamoto, Japan) for help with the immunostaining and direct sequencing.

\section{References}

1. Roth JG and Elvidge AR: Glioblastoma multiforme: a clinical survey. J Neurosurg 17: 736-750, 1960.

2. Grahovac G, Tomac D, Lambasa S, et al: Cerebellar glioblastomas: pathophysiology, clinical presentation and management. Acta Neurochir (Wien) 151: 653-657, 2009.

3. Salazar OM: Primary malignant cerebellar astrocytomas in children: a signal for postoperative craniospinal irradiation. Int J Radiat Oncol Biol Phys 7: 1661-1665, 1981.

4. Kleihues $\mathrm{P}$ and Ohgaki H: Primary and secondary glioblastomas: from concept to clinical diagnosis. Neuro-Oncol 1: 44-51, 1999

5. Ohgaki $\mathrm{H}$ and Kleihues P: Genetic pathways to primary and secondary glioblastoma. Am J Pathol 170: 1445-1453, 2007
6. Nobusawa S, Watanabe T, Kleihues $\mathrm{P}$, et al: IDH1 mutations as molecular signature and predictive factor of secondary glioblastomas. Clin Cancer Res 15: 6002-6007, 2009.

7. Weber DC, Miller RC, Villà S, et al: Outcome and prognostic factors in cerebellar glioblastoma multiforme in adults: a retrospective study from the rare cancer network. Int J Radiat Oncol Biol Phys 66: 179-186, 2006.

8. Tsung AJ, Prabhu SS, Lei X, Chern JJ, Benjamin Bekele N and Shonka NA: Cerebellar glioblastoma: a retrospective review of 21 patients at a single institution. J Neurooncol 105: 555-562, 2011

9. Lammert M, Friedman JM, Kluwe L, et al: Prevalance of neurofibromatosis 1 in German children at elementary school enrollment. Arch Dermatol 141: 71-74, 2005.

10. Easton DF, Ponder SM, Huson SM, et al: An analysis of variation in expression of neurofibromatosis (NF) type 1 (NF1): evidence for modifying genes. Am J Hum Genet 53: 305-313, 1993.

11. Brown PD, Buckner JC, O'Fallon JR, et al: Adult patients with supratentorial pilocytic astrocytomas: a prospective multicenter clinical trial. Int J Radiat Oncol Biol Phys 58: 1153-1160, 2004.

12. Canesso A, Gardiman M, Salmaso R, et al: An unusual case of malignant pilocytic astrocytoma occurring in the eye. Virchows Arch 449: 248-252, 2006.

13. Gilles FH, Sobel EL, Tavaré CJ, et al: Age-related changes in diagnoses, histological features, and survival in children with brain tumors: 1930-1979. The Childhood Brain Tumor Consortium. Neurosurgery 37: 1056-1068, 1995.

14. Roessler K, Bertalanffy A, Jezan H, et al: Proliferative activity as measured by MIB-1 labeling index and long-term outcome of cerebellar juvenile pilocytic astrocytomas. J Neurooncol 58: 141-146, 2002.

15. Cawthon RM, Weiss R, Xu GF, et al: A major segment of the neurofibromatosis type 1 gene: cDNA sequence, genomic structure, and point mutations. Cell 62: 193-201, 1990.

16. Xu GF, Lin B, Tanaka K, et al: The catalytic domain of the neurofibromatosis type 1 gene product stimulates ras GTPase and complements ira mutants of S.cerevisiae. Cell 63: 835-841, 1990.

17. Zhu Y, Guignard F, Zhao D, et al: Early inactivation of p53 tumor suppressor gene cooperating with NF1 loss induces malignant astrocytoma. Cancer Cell 8: 119-130, 2005.

18. Klériga E, Sher JH, Nallainathan SK, et al: Development of cerebellar malignant astrocytoma at site of a medulloblastoma treated 11 years earlier. Case report. J Neurosurg 49: 445-449, 1978.

19. Cohen MS, Kushner MJ and Dell S: Frontal lobe astrocytoma following radiotherapy for medulloblastoma. Neurology 31 : 616-619, 1981.

20. Furuta T, Sugiu K, Tamiya T, et al: Malignant cerebellar astrocytoma developing 15 years after radiation therapy for a medulloblastoma. Clin Neurol Neurosurg 100: 56-59, 1998.

21. Nakamizo A, Nishio S, Inamura T, et al: Evolution of malignant cerebellar astrocytoma at the site of a treated medulloblastoma: report of two cases. Acta Neurochir (Wien) 143: 697-700, 2001.

22. Osumi AK, Mclendon RE, Tien RD, et al: Well differentiated astrocytoma occurring nine years after radiation therapy for medulloblastoma. Clin Neuropathol 13: 281-285, 1994.

23. Pearl GS, Mirra SS and Miles ML: Glioblastoma multiforme occurring 13 years after treatment of a medulloblastoma. Neurosurgery 6: 546-551, 1980.

24. Safneck JR, Napier LB and Halliday WC: Malignant astrocytoma of the optic nerve in a child. Can J Neurol Sci 19: 498-503, 1992.

25. Schmidbauer M, Budka H, Bruckner R, et al: Glioblastoma developing at the site of a cerebellar medulloblastoma treated 6 years earlier. Case report. J Neurosurg 67: 915-918, 1987.

26. Paulino AC, Mai WY, Chintagumpala M, et al: Radiation-induced malignant gliomas: is there a role for reirradiation? Int J Radiat Oncol Biol Phys 71: 1381-1387, 2008.

27. Utsuki S, Oka H, Miyajima Y, et al: Adult cerebellar glioblastoma cases have different characteristics from supratentorial glioblastoma. Brain Tumor Pathol 29: 87-95, 2012.

28. Saito T, Hama S, Kajiwara Y, et al: Prognosis of cerebellar glioblastomas: correlation between prognosis and immunoreactivity for epidermal growth factor receptor compared with supratentorial glioblastomas. Anticancer Res 26: 1351-1357, 2006.

29. Akimoto J, Fukami S, Tsutsumi M, et al: Radiopathological characteristics of cerebellar malignant glioma in adults. Brain Tumor Pathol 26: 59-68, 2009.

30. Balss J, Meyer J, Mueller W, et al: Analysis of the IDH1 codon 132 mutation in brain tumors. Acta Neuropathol 116: 597-602, 2008. 
31. Gravendeel LA, Kloosterhof NK, Bralten LB, et al: Segregation of non-p.R132H mutations in IDH1 in distinct molecular subtypes of glioma. Hum Mutat 31: E1186-E1190, 2010.

32. Ichimura K, Pearson DM, Kocialkowski S, et al: IDH1 mutations are present in the majority of common adult gliomas but are rare in primary glioblastomas. Neuro Oncol 11: 341-347, 2009.

33. Sanson M, Marie Y, Paris S, et al: Isocitrate dehydrogenase 1 codon 132 mutation is an important prognostic biomarker in gliomas. J Clin Oncol 27: 4150-4154, 2009.

34. Sonoda Y, Kumabe T, Nakamura T, et al: Analysis of IDH1 and IDH2 mutations in Japanese glioma patients. Cancer Sci 100: 1996-1998, 2009.
35. van den Bent MJ, Dubbink HJ, Marie Y, et al: IDH1 and IDH2 mutations are prognostic but not predictive for outcome in anaplastic oligodendroglial tumors: a report of the European Organization for Research and Treatment of Cancer Brain Tumor Group. Clin Cancer Res 16: 1597-1604, 2010

36. Watanabe T, Nobusawa S, Kleihues $\mathrm{P}$, et al: IDH1 mutations are early events in the development of astrocytomas and oligodendrogliomas. Am J Pathol 174: 1149-1153, 2009.

37. Weller M, Felsberg J, Hartmann C, et al: Molecular predictors of progression-free and overall survival in patients with newly diagnosed glioblastoma: a prospective translational study of the German Glioma Network. J Clin Oncol 27: 5743-5750, 2009. 